CRYSTALLOGRAPHIC COMMUNICATIONS

ISSN 2056-9890

Received 23 July 2017

Accepted 4 August 2017

Edited by $\mathrm{H}$. Stoeckli-Evans, University of Neuchâtel, Switzerland

Keywords: crystal structure; anthracene; indole; Schiff base; tryptamine; methanamine; $\mathrm{N}$ $\mathrm{H} \cdots \pi$ interactions; $\mathrm{C}-\mathrm{H} \cdots \pi$ interactions.

CCDC reference: 1537222

Supporting information: this article has supporting information at journals.iucr.org/e

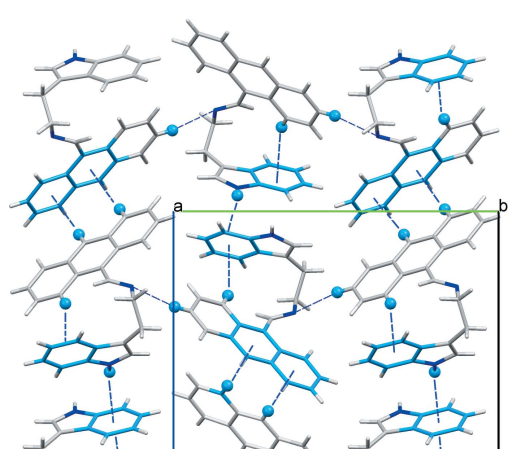

OPEN $\odot$ ACCESS

\section{Crystal structure and DFT study of $(E)-\mathrm{N}-[2-(1 \mathrm{H}-$ indol-3-yl)ethyl]-1-(anthracen-9-yl)methanimine}

\author{
Md. Serajul Haque Faizi, ${ }^{a}$ Necmi Dege, ${ }^{b}$ Sergey Malinkin ${ }^{c}$ and Tetyana Yu. Sliva ${ }^{\mathrm{c} *}$ \\ a Department of Chemistry, College of Science, Sultan Qaboos University, PO Box 36 Al-Khod 123, Muscat, Sultanate of \\ Oman, 'bondokuz Mayıs University, Arts and Sciences Faculty, Department of Physics, 55139 Atakum-Samsun, Turkey, \\ and ${ }^{\mathbf{C}}$ Department of Chemistry, National Taras Shevchenko University of Kiev, 64/13 Volodymyrska Street, City of Kyiv \\ 01601, Ukraine. *Correspondence e-mail: malinachem88@gmail.com
}

The title compound, $\mathrm{C}_{25} \mathrm{H}_{20} \mathrm{~N}_{2}$, (I), was synthesized from the condensation reaction of anthracene-9-carbaldehyde and tryptamine in dry ethanol. The indole ring system (r.m.s. deviation $=0.016 \AA$ ) makes a dihedral angle of $63.56(8)^{\circ}$ with the anthracene ring (r.m.s. deviation $=0.023 \AA$ ). There is a short intramolecular $\mathrm{C}-\mathrm{H} \cdots \mathrm{N}$ interaction present, and a $\mathrm{C}-\mathrm{H} \cdots \pi$ interaction involving the two ring systems. In the crystal, the indole $\mathrm{H}$ atom forms an intermolecular $\mathrm{N}-\mathrm{H} \cdots \pi$ interaction, linking molecules to form chains along the $b$-axis direction. There are also $\mathrm{C}-\mathrm{H} \cdots \pi$ interactions present, involving the central and terminal rings of the anthracene unit, linking the chains to form an overall two-dimensional layered structure, with the layers parallel to the $b c$ plane. The density functional theory (DFT) optimized structure, at the B3LYP/ 6-311 G(d,p) level, is compared with the experimentally determined molecular structure in the solid state.

\section{Chemical context}

Tryptamine is a biogenic serotonin-related indoamine and is the decarboxylation product of the amino acid tryptophan. $2-(1 H$-Indol-3-yl)ethanamine is an alkaloid found in plants and fungi and is a possible intermediate in the biosynthetic pathway to the plant hormone indole-3-acetic acid (Takahashi, 1986). It is also found in trace amounts in the mammalian brain, possibly acting as a neuromodulator or neurotransmitter (Jones, 1982). There are seven known families of serotonin receptors, which are tryptamine derivatives. All of them are neurotransmitters. Hallucinogens all have a high affinity for certain serotonin receptor subtypes and the relative hallucinogenic potencies of various drugs can be gauged by their affinities for these receptors (Glennon et al., 1984; Nichols \& Sanders-Bush, 2001; Johnson et al., 1987; KrebsThomson et al., 1998). The structures of many hallucinogens are similar to serotonin and have a tryptamine core. Indole analogues, especially of tryptamine derivatives, have been found to be polyamine site antagonists at the $N$-methyl-Daspartate receptor (NMDAR; Worthen et al., 2001). Indole and its derivatives are secondary metabolites that are present in most plants (such as unripe bananas, broccoli and clove), almost all flower oils (e.g. jasmine and orange blossoms) and coal tar (Waseem \& Mark 2005; Lee et al., 2003). In the pharmaceutical field, it has been discovered that it has antimicrobial and anti-inflammatory properties (Mohammad \& Moutaery, 2005). The present work is part of an ongoing structural study of Schiff bases and their utilization in the synthesis of new organic and polynuclear coordination compounds, and their application in fluorescence sensors 
(Faizi \& Sen, 2014; Faizi et al., 2016). We report herein the crystal structure of $(E)-N$-[2-(1H-indol-3-yl)ethyl]-1-(anthracen-9-yl)methanimine, (I), and its DFT computational calculation. Calculations by density functional theory (DFT) on (I), carried out at the B3LYP/6-311 G(d,p) level, are compared with the experimentally determined molecular structure in the solid state.

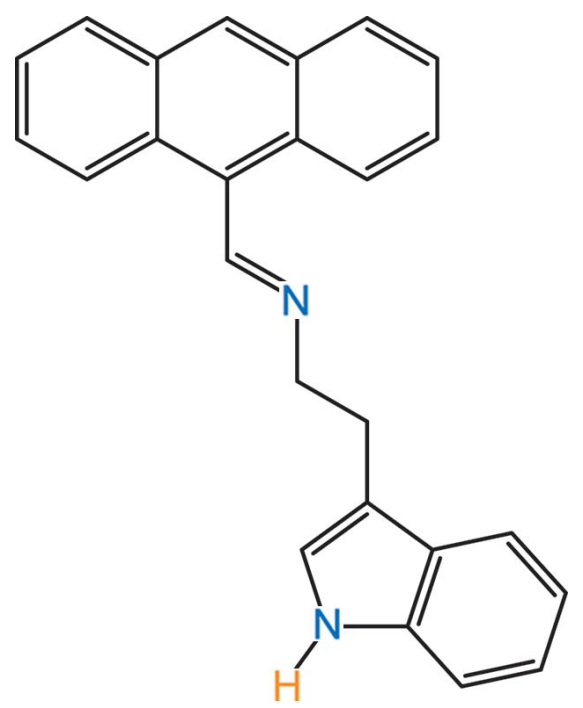

\section{Structural commentary}

The molecular structure of compound (I) is illustrated in Fig. 1. The molecule adopts a nonplanar geometry, with the dihedral angle between the planes of the indole and anthracene rings being $63.56(8)^{\circ}$. The conformation about the azomethine $\mathrm{C} 15=\mathrm{N} 1$ bond $[1.272(10) \AA]$ is $E$, with the $\mathrm{C} 14-\mathrm{N} 2-\mathrm{C} 12-$ C13 torsion angle being $179.0(1)^{\circ}$. The molecule is stabilized by a weak intramolecular hydrogen bond $(\mathrm{C} 12-\mathrm{H} 12 \cdots \mathrm{N} 1)$

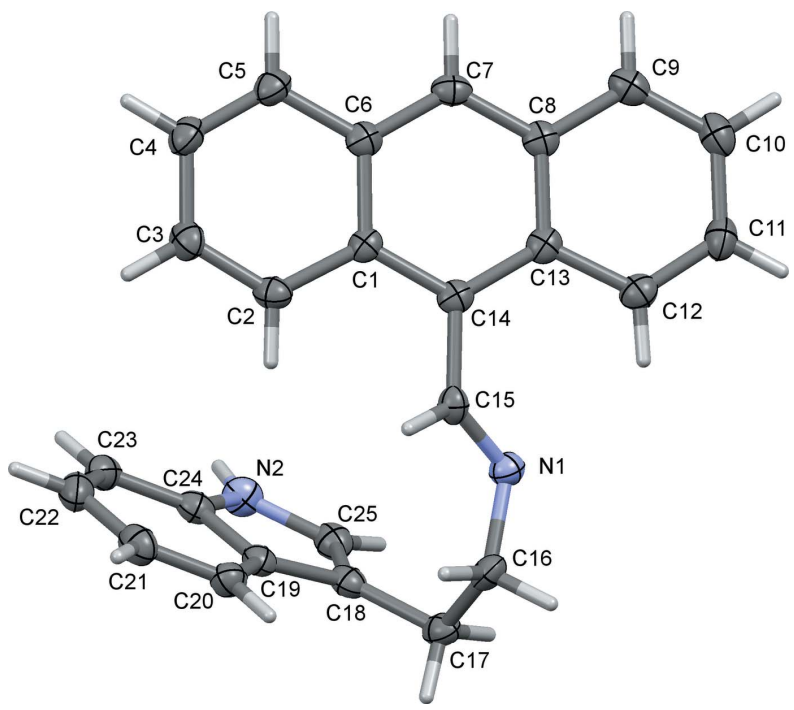

Figure 1

The molecular structure of compound (I), with the atom labelling. Displacement ellipsoids are drawn at the $50 \%$ probability level.
Table 1

Hydrogen-bond geometry $\left(\AA,^{\circ}\right)$.

$\mathrm{Cg} 3, \mathrm{Cg} 4$ and $\mathrm{Cg} 5$ are the centroids rings $\mathrm{C} 1 / \mathrm{C} 6-\mathrm{C} 8 / \mathrm{C} 13 / \mathrm{C} 14, \mathrm{C} 8-\mathrm{C} 13$ and C19-C24, respectively.

\begin{tabular}{lllll}
\hline$D-\mathrm{H} \cdots A$ & $D-\mathrm{H}$ & $\mathrm{H} \cdots A$ & $D \cdots A$ & $D-\mathrm{H} \cdots A$ \\
\hline $\mathrm{C} 12-\mathrm{H} 12 \cdots \mathrm{N} 1$ & 0.93 & 2.36 & $2.9845(2)$ & 124 \\
$\mathrm{C} 2-\mathrm{H} 2 \cdots C g 5$ & 0.93 & 2.77 & $3.5505(2)$ & 142 \\
$\mathrm{~N} 2-\mathrm{H} 2 A \cdots C g 5^{\mathrm{i}}$ & 0.86 & 2.59 & $3.1855(2)$ & 127 \\
$\mathrm{C} 7-\mathrm{H} 7 \cdots C g 4^{\mathrm{ii}}$ & 0.93 & 2.75 & $3.5777(2)$ & 148 \\
$\mathrm{C} 9-\mathrm{H} 9 \cdots C g 3^{\mathrm{ii}}$ & 0.93 & 2.73 & $3.5077(2)$ & 142 \\
$\mathrm{C} 16-\mathrm{H} 16 A \cdots C g 3^{\mathrm{iii}}$ & 0.97 & 2.86 & $3.5375(2)$ & 128 \\
\hline
\end{tabular}

Symmetry codes: (i) $x-\frac{1}{2},-y+\frac{1}{2},-z$; (ii) $x-\frac{1}{2},-y+\frac{1}{2},-z+1$; (iii) $x+1, y, z$.

and a $\mathrm{C}-\mathrm{H} \cdots \pi$ interaction $(\mathrm{C} 2-\mathrm{H} 2 \cdots C g 5 ; C g 5$ is the centroid of the C19-C24 ring); see Table 1.

\section{Supramolecular features}

In the crystal, the indole $\mathrm{H}$ atom forms an intermolecular $\mathrm{N}-$ $\mathrm{H} \cdots \pi$ interaction, linking molecules to form chains along the $b$-axis direction (Fig. 2 and Table 1). There are also $\mathrm{C}-\mathrm{H} \cdots \pi$ interactions present, involving the central ring and terminal rings of the anthracene unit, linking the chains to form layers parallel to the $b c$ plane (Fig. 2 and Table 1).

\section{Database survey}

A search of the Cambridge Structural Database (CSD, Version 5.38, update February 2017; Groom et al., 2016)

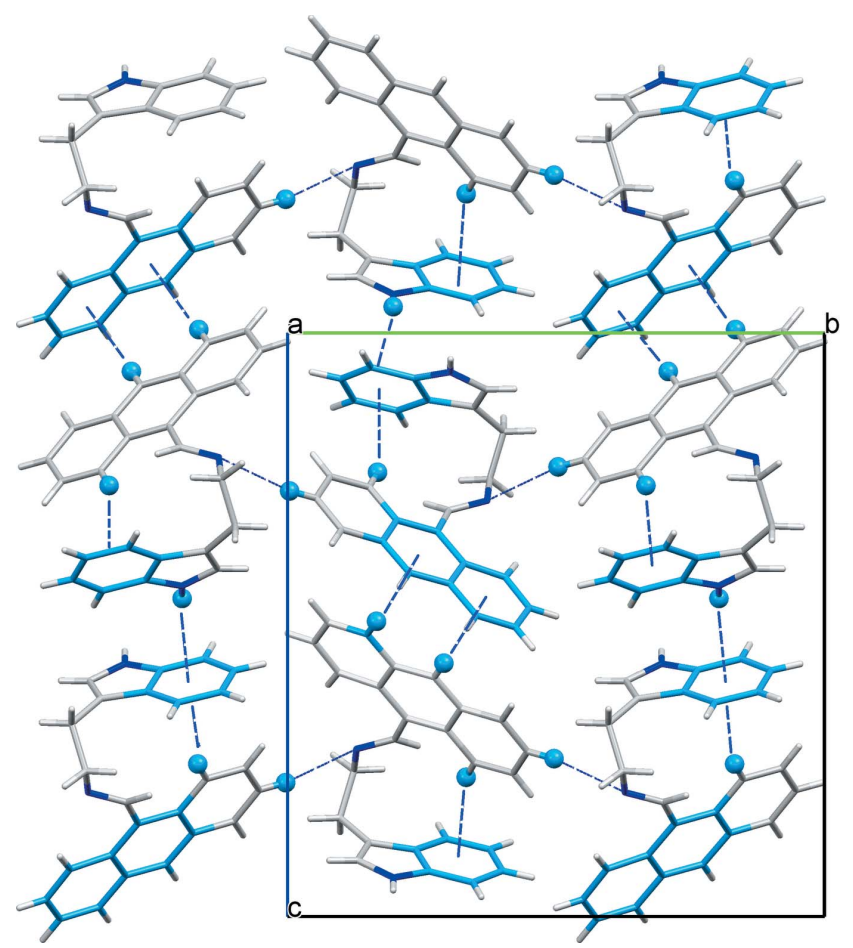

Figure 2

A view along the $a$ axis of the crystal packing of compound (I), showing the layer-like structure. Weak $\mathrm{N}-\mathrm{H} \cdots \pi$ and $\mathrm{C}-\mathrm{H} \cdots \pi$ interactions are shown as blue dashed lines (see Table 1). 
Table 2

Comparison of selected geometric data for $(\mathbf{I})\left(\AA{ }^{\circ}\right)$ from X-ray and calculated (DFT) data.

\begin{tabular}{lll}
\hline & X-ray & B3LYP/6-311G(d,p) \\
\hline $\mathrm{N} 1-\mathrm{C} 15$ & $1.272(3)$ & 1.271 \\
$\mathrm{~N} 1-\mathrm{C} 16$ & $1.468(4)$ & 1.466 \\
$\mathrm{C} 16-\mathrm{C} 17$ & $1.528(4)$ & 1.531 \\
$\mathrm{C} 17-\mathrm{C} 18$ & $1.499(4)$ & 1.494 \\
$\mathrm{C} 15-\mathrm{C} 14$ & $1.479(4)$ & 1.494 \\
$\mathrm{C} 25-\mathrm{N} 2$ & $1.372(3)$ & 1.369 \\
$\mathrm{~N} 2-\mathrm{C} 24$ & $1.371(4)$ & 1.371 \\
$\mathrm{C} 16-\mathrm{N} 1-\mathrm{C} 15$ & $115.2(2)$ & 115.31 \\
$\mathrm{~N} 1-\mathrm{C} 16-\mathrm{C} 17$ & $110.4(2)$ & 110.50 \\
$\mathrm{~N} 1-\mathrm{C} 15-\mathrm{C} 14$ & $126.3(3)$ & 126.16 \\
$\mathrm{C} 16-\mathrm{C} 17-\mathrm{C} 18$ & $112.2(2)$ & 112.27 \\
\hline
\end{tabular}

revealed the structures of several similar compounds containing a phenol group [(II) (CSD refcode FAJVIV; Rodriguez et al., 1987) and (III) (TANNOL; Ishida et al., 1992)] and nitrobenzene moieties [(IV), GEYPEF; Törnroos, 1988]. All compounds are 2-indole-substituted derivatives which have two aromatic units linked via an aliphatic chain. In (I), the dihedral angle between indole and anthracene rings is $63.56(8)^{\circ}$, which is similar for (III) and (IV), viz. 71.52 and $64.21^{\circ}$, respectively. In compounds (I) and (II), the conformation about the azomethine $\mathrm{C} 15=\mathrm{N} 1$ bond is $E$.

\section{DFT study}

Calculations by density functional theory DFT-B3LYP, with basis set 6-311 G(d,p), of bond lengths and angles were performed. These values are compared with the experimental values for the title system (see Table 2). From these results we can conclude that basis set $6-311 \mathrm{G}(\mathrm{d}, \mathrm{p})$ is better suited in its approach to the experimental data.

The LUMO and HOMO orbital energy parameters are considerably answerable for the charge transfer, chemical reactivity and kinetic/thermodynamic stability of (I). The DFT study of (I) revealed that the HOMO and LUMO are localized in the plane extending from the whole anthracene ring to the indole ring, and electron distribution of the HOMO-1, HOMO, LUMO and LUMO+1 energy levels are shown in Fig. 3. The molecular orbital of HOMO contains both $\sigma$ and $\pi$ character, whereas HOMO-1 is dominated by $\pi$-orbital density. The LUMO is mainly composed of $\sigma$ density, while LUMO +1 has both $\sigma$ and $\pi$ electronic density. The HOMOLUMO gap for (I) was found to be 0.12325 a.u. and the frontier molecular orbital energies, $E_{\mathrm{HOMO}}$ and $E_{\mathrm{LUMO}}$, were found to be -0.196412 and -0.07087 a.u., respectively.

\section{Synthesis and crystallization}

$80 \mathrm{mg}(0.435 \mathrm{mmol})$ of $2-(1 H$-indol-3-yl)ethanamine (tryptamine) were dissolved in $10 \mathrm{ml}$ of absolute ethanol. To this solution, $89 \mathrm{mg}$ (0.434 $\mathrm{mmol})$ of anthracene-9-carbaldehyde in $5 \mathrm{ml}$ of absolute ethanol were added dropwise under stirring. The mixture was stirred for $10 \mathrm{~min}$, two drops of glacial acetic acid were added and the mixture was refluxed for a further $2 \mathrm{~h}$.
The resulting yellow precipitate was recovered by filtration, washed several times with small portions of ice-cold ethanol and then with diethyl ether to give $140 \mathrm{mg}$ (87\%) of compound (I). Dark-yellow block-like crystals suitable for $\mathrm{X}$-ray analysis were obtained within $3 \mathrm{~d}$ by slow evaporation of a solution in methanol.

\section{Refinement}

Crystal data, data collection and structure refinement details are summarized in Table 3 . The $\mathrm{N}-\mathrm{H} \mathrm{H}$ atom was located from a difference-Fourier map and constrained to ride on the parent atom: $\mathrm{N}-\mathrm{H}=0.86 \AA$ and $U_{\text {iso }}(\mathrm{H})=1.2 U_{\text {eq }}(\mathrm{N})$. All Cbound $\mathrm{H}$ atoms were positioned geometrically and refined using a riding model, with $\mathrm{C}-\mathrm{H}=0.93-0.97 \AA$ and $U_{\text {iso }}(\mathrm{H})=$ $1.2 U_{\text {eq }}(\mathrm{C})$.

The DFT quantum-chemical calculations were performed at the B3LYP/6-311 G(d,p) level (Becke, 1993; Lee et al., 2003) as implemented in GAUSSIAN09 (Frisch et al., 2009). DFT structure optimization of (I) was performed starting from the $\mathrm{X}$-ray geometry.
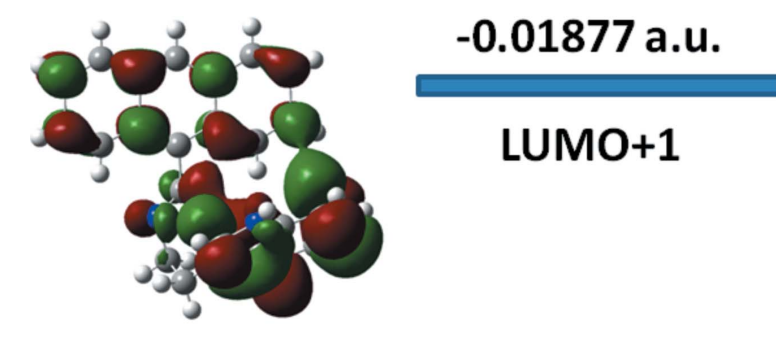

LUMO+1

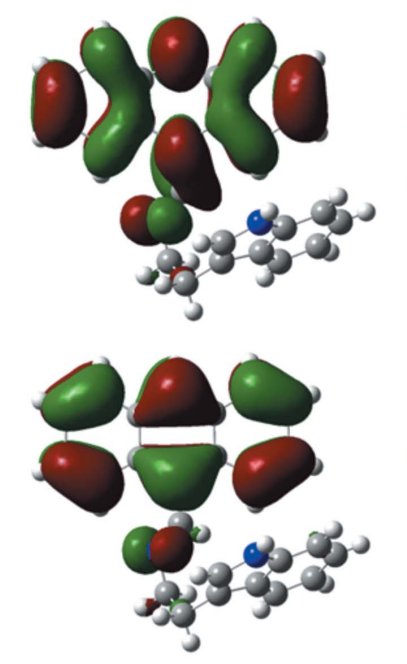

-0.07087 a.u.

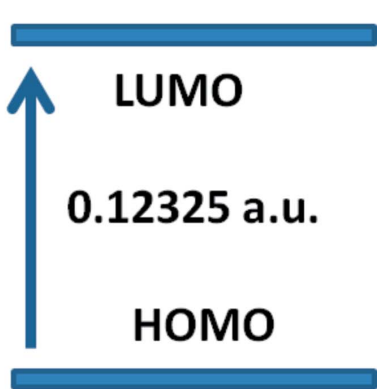

-0.19412 a.u.

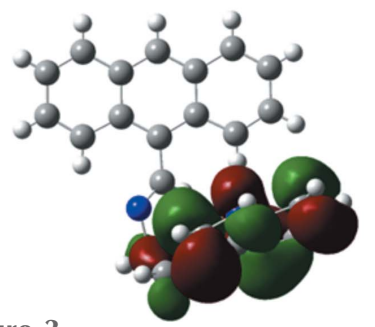

HOMO-1

Figure 3

Electron distribution of the HOMO-1, HOMO, LUMO and LUMO+1 energy levels for compound (I). 


\section{Acknowledgements}

The authors are grateful to the Ondokuz Mayıs University, Arts and Sciences Faculty, Department of Physics, Turkey, for $\mathrm{X}$-ray data collection, and Department of Chemistry, National Taras Shevchenko University of Kiev.

\section{References}

Becke, A. D. (1993). J. Chem. Phys. 98, 5648-5652.

Bruker (2003). SMART, SAINT and SADABS. Bruker AXS Inc., Madison, Wisconsin, USA.

Faizi, M. S. H., Gupta, S., Mohan, V. K., Jain, K. V. \& Sen, P. (2016). Sens. Actuators B, 222, 15-20.

Faizi, M. S. H. \& Sen, P. (2014). Acta Cryst. E70, m206-m207.

Frisch, M. J., et al. (2009). GAUSSIAN09. Gaussian Inc., Wallingford, CT, USA.

Glennon, R. A., Titeler, M. \& McKenney, J. D. (1984). Life Sci. 35, 2505-2511.

Groom, C. R., Bruno, I. J., Lightfoot, M. P. \& Ward, S. C. (2016). Acta Cryst. B72, 171-179.

Ishida, T., Negi, A., In, Y. \& Inoue, M. (1992). Acta Cryst. C48, 193194.

Johnson, M. P., Hoffman, A. J., Nichols, D. E. \& Mathis, C. A. (1987). Neuropharmacology, 26, 1803-1806.

Jones, R. S. G. (1982). Prog. Neurobiol. 19, 117-139.

Krebs-Thomson, K., Paulus, M. P. \& Geyer, M. A. (1998). Neuropsychopharmacology, 18, 339-351.

Lee, S. K., Yi, K. Y., Kim, S. K., Suh, J., Kim, N. J., Yoo, S., Lee, B. H., Seo, H. W., Kim, S. O. \& Lim, H. (2003). Eur. J. Med. Chem. 38, 459-471.

Macrae, C. F., Bruno, I. J., Chisholm, J. A., Edgington, P. R., McCabe, P., Pidcock, E., Rodriguez-Monge, L., Taylor, R., van de Streek, J. \& Wood, P. A. (2008). J. Appl. Cryst. 41, 466-470.

Mohammad, T. \& Moutaery, A. A. (2005). Exp. Tox. Path. 56, 119129.

Nichols, C. D. \& Sanders-Bush, E. (2001). Heffter Rev. Psychedelic Res. 2, 73-79.

Rodriguez, M. L., Medina de la Rosa, E., Gili, P., Zarza, P. M., Reyes, M. G. M., Medina, A. \& Díaz González, M. C. (1987). Acta Cryst. C43, 134-136.
Table 3

Experimental details.

\begin{tabular}{ll}
\hline Crystal data & \\
Chemical formula & $\mathrm{C}_{25} \mathrm{H}_{20} \mathrm{~N}_{2}$ \\
$M_{\mathrm{r}}$ & 348.43 \\
Crystal system, space group & Orthorhombic, $P 2_{1} 2_{1} 2_{1}$ \\
Temperature $(\mathrm{K})$ & 100 \\
$a, b, c(\AA)$ & $6.0044(3), 16.4721(7), 17.8957(9)$ \\
$V\left(\AA^{3}\right)$ & $1769.98(15)$ \\
$Z$ & 4 \\
Radiation type & Mo $K \alpha$ \\
$\mu\left(\mathrm{mm}^{-1}\right)$ & 0.08 \\
Crystal size $(\mathrm{mm})$ & $0.20 \times 0.15 \times 0.13$ \\
& \\
Data collection & Bruker SMART APEX CCD \\
Diffractometer & Multi-scan $(S A D A B S ;$ Bruker, \\
Absorption correction & $2003)$ \\
& $0.875,0.990$ \\
$T_{\min }, T_{\max }$ & $14169,3127,2577$ \\
No. of measured, independent and & \\
$\quad$ observed $[I>2 \sigma(I)]$ reflections & 0.064 \\
$R_{\text {int }}$ & 0.596 \\
(sin $\theta / \lambda)_{\text {max }}\left(\AA^{-1}\right)$ & \\
Refinement & \\
$R\left[F^{2}>2 \sigma\left(F^{2}\right)\right], w R\left(F^{2}\right), S$ & $0.041,0.093,1.04$ \\
No. of reflections & 3127 \\
No. of parameters & 245 \\
H-atom treatment & H-atom parameters constrained \\
$\Delta \rho_{\text {max }}, \Delta \rho_{\min }\left(\mathrm{e} \AA^{-3}\right)$ & $0.15,-0.20$ \\
Absolute structure & Refined as an inversion twin \\
\hline
\end{tabular}

Computer programs: SMART and SAINT (Bruker, 2003), SHELXT (Sheldrick, 2015a), SHELXTL (Sheldrick, 2008), Mercury (Macrae et al., 2008), SHELXL2016 (Sheldrick, 2015b) and PLATON (Spek, 2009).

Sheldrick, G. M. (2008). Acta Cryst. A64, 112-122.

Sheldrick, G. M. (2015a). Acta Cryst. A71, 3-8.

Sheldrick, G. M. (2015b). Acta Cryst. C71, 3-8.

Spek, A. L. (2009). Acta Cryst. D65, 148-155.

Takahashi, N. (1986). In Chemistry of Plant Hormones. Florida: CRC Press.

Törnroos, K. W. (1988). Acta Cryst. C44, 1238-1240.

Waseem, G. \& Mark, T. H. (2005). Life Sci. 78, 442-453.

Worthen, D. R., Gibson, D. A., Rogers, D. T., Bence, A. K., Fu, M., Littleton, J. M. \& Crooks, P. A. (2001). Brain Res. 890, 343-346. 


\section{supporting information}

Acta Cryst. (2017). E73, 1329-1332 [https://doi.org/10.1107/S2056989017011483]

Crystal structure and DFT study of $(E)-N-[2-(1 /$-indol-3yl)ethyl]-1-(anthracen-9-yl)methanimine

Md. Serajul Haque Faizi, Necmi Dege, Sergey Malinkin and Tetyana Yu. Sliva

Computing details

Data collection: SMART (Bruker, 2003); cell refinement: SAINT (Bruker, 2003); data reduction: SAINT (Bruker, 2003);

program(s) used to solve structure: SHELXT (Sheldrick, 2015a); program(s) used to refine structure: SHELXL2016

(Sheldrick, 2015b); molecular graphics: SHELXTL (Sheldrick, 2008) and Mercury (Macrae et al., 2008); software used to prepare material for publication: SHELXL2016 (Sheldrick, 2015b) and PLATON (Spek, 2009).

(E)-N-[2-(1H-Indol-3-yl)ethyl]-1-(anthracen-9-yl)methanimine

Crystal data

$\mathrm{C}_{25} \mathrm{H}_{20} \mathrm{~N}_{2}$

$M_{r}=348.43$

Orthorhombic, $P 22_{1} 2_{1} 2_{1}$

$a=6.0044(3) \AA$

$b=16.4721(7) \AA$

$c=17.8957$ (9) $\AA$

$V=1769.98(15) \AA^{3}$

$Z=4$

$F(000)=736$

Data collection

Bruker SMART APEX CCD

diffractometer

Radiation source: fine-focus sealed tube

Graphite monochromator

$\omega$ scans

Absorption correction: multi-scan

(SADABS; Bruker, 2003)

$T_{\min }=0.875, T_{\max }=0.990$

Refinement

Refinement on $F^{2}$

Least-squares matrix: full

$R\left[F^{2}>2 \sigma\left(F^{2}\right)\right]=0.041$

$w R\left(F^{2}\right)=0.093$

$S=1.04$

3127 reflections

245 parameters

0 restraints

Primary atom site location: structure-invariant direct methods
$D_{\mathrm{x}}=1.308 \mathrm{Mg} \mathrm{m}^{-3}$

Mo $K \alpha$ radiation, $\lambda=0.71073 \AA$

Cell parameters from 5148 reflections

$\theta=2.6-27.4^{\circ}$

$\mu=0.08 \mathrm{~mm}^{-1}$

$T=100 \mathrm{~K}$

Needle, yellow

$0.20 \times 0.15 \times 0.13 \mathrm{~mm}$

14169 measured reflections

3127 independent reflections

2577 reflections with $I>2 \sigma(I)$

$R_{\text {int }}=0.064$

$\theta_{\max }=25.1^{\circ}, \theta_{\min }=2.3^{\circ}$

$h=-7 \rightarrow 7$

$k=-19 \rightarrow 19$

$l=-21 \rightarrow 21$

Secondary atom site location: difference Fourier map

Hydrogen site location: inferred from neighbouring sites

$\mathrm{H}$-atom parameters constrained

$w=1 /\left[\sigma^{2}\left(F_{\mathrm{o}}^{2}\right)+(0.0478 P)^{2}\right]$

where $P=\left(F_{\mathrm{o}}{ }^{2}+2 F_{\mathrm{c}}{ }^{2}\right) / 3$

$(\Delta / \sigma)_{\max }<0.001$

$\Delta \rho_{\max }=0.15$ e $\AA^{-3}$

$\Delta \rho_{\text {min }}=-0.20$ e $\AA^{-3}$

Absolute structure: Refined as an inversion twin 


\section{Special details}

Geometry. All esds (except the esd in the dihedral angle between two 1.s. planes) are estimated using the full covariance matrix. The cell esds are taken into account individually in the estimation of esds in distances, angles and torsion angles; correlations between esds in cell parameters are only used when they are defined by crystal symmetry. An approximate (isotropic) treatment of cell esds is used for estimating esds involving l.s. planes.

Fractional atomic coordinates and isotropic or equivalent isotropic displacement parameters $\left(\AA^{2}\right)$

\begin{tabular}{|c|c|c|c|c|}
\hline & $x$ & $y$ & $z$ & $U_{\text {iso }} * / U_{\text {eq }}$ \\
\hline N1 & $0.9441(4)$ & $0.37168(13)$ & $0.28719(12)$ & $0.0239(6)$ \\
\hline $\mathrm{N} 2$ & $0.6749(4)$ & $0.30313(14)$ & $0.06673(12)$ & $0.0282(6)$ \\
\hline $\mathrm{H} 2 \mathrm{~A}$ & 0.546902 & 0.303463 & 0.045136 & $0.034^{*}$ \\
\hline $\mathrm{C} 18$ & $0.9807(5)$ & $0.34812(16)$ & $0.12665(15)$ & $0.0239(7)$ \\
\hline $\mathrm{C} 1$ & $0.5718(5)$ & $0.20309(15)$ & $0.32067(14)$ & $0.0199(6)$ \\
\hline $\mathrm{C} 8$ & $0.3608(5)$ & $0.30172(16)$ & $0.42939(14)$ & $0.0216(6)$ \\
\hline $\mathrm{C} 15$ & $0.8796(5)$ & $0.30043(16)$ & $0.30408(14)$ & $0.0219(6)$ \\
\hline H15 & 0.975892 & 0.257990 & 0.292647 & $0.026^{*}$ \\
\hline $\mathrm{C} 13$ & $0.5640(5)$ & $0.32872(15)$ & $0.39461(15)$ & $0.0216(6)$ \\
\hline C14 & $0.6662(5)$ & $0.27861(15)$ & $0.34017(14)$ & $0.0206(6)$ \\
\hline $\mathrm{C} 7$ & $0.2732(5)$ & $0.22659(16)$ & $0.41038(14)$ & $0.0235(7)$ \\
\hline $\mathrm{H} 7$ & 0.144366 & 0.208937 & 0.434185 & $0.028^{*}$ \\
\hline C9 & $0.2567(5)$ & $0.35163(17)$ & $0.48380(15)$ & $0.0265(7)$ \\
\hline H9 & 0.124712 & 0.334465 & 0.505954 & $0.032 *$ \\
\hline C19 & $1.0013(5)$ & $0.26179(15)$ & $0.11461(15)$ & $0.0227(7)$ \\
\hline C6 & $0.3713(5)$ & $0.17673(16)$ & $0.35700(14)$ & $0.0226(7)$ \\
\hline $\mathrm{C} 2$ & $0.6601(5)$ & $0.15155(15)$ & $0.26395(14)$ & $0.0247(7)$ \\
\hline $\mathrm{H} 2$ & 0.788158 & 0.167424 & 0.238604 & $0.030^{*}$ \\
\hline $\mathrm{C} 5$ & $0.2749(5)$ & $0.10058(16)$ & $0.33667(15)$ & $0.0274(7)$ \\
\hline H5 & 0.146320 & 0.083137 & 0.360777 & $0.033^{*}$ \\
\hline $\mathrm{C} 20$ & $1.1624(5)$ & $0.20388(17)$ & $0.13290(15)$ & $0.0256(7)$ \\
\hline $\mathrm{H} 20$ & 1.293314 & 0.219244 & 0.156804 & $0.031^{*}$ \\
\hline $\mathrm{C} 24$ & $0.8068(5)$ & $0.23616(17)$ & $0.07725(15)$ & $0.0250(7)$ \\
\hline $\mathrm{C} 23$ & $0.7688(5)$ & $0.15516(17)$ & $0.05852(15)$ & $0.0267(7)$ \\
\hline $\mathrm{H} 23$ & 0.639991 & 0.139260 & 0.033642 & $0.032 *$ \\
\hline $\mathrm{C} 3$ & $0.5619(5)$ & $0.07970(16)$ & $0.24594(16)$ & $0.0276(7)$ \\
\hline $\mathrm{H} 3$ & 0.624024 & 0.047503 & 0.208676 & $0.033^{*}$ \\
\hline $\mathrm{C} 4$ & $0.3669(5)$ & $0.05321(16)$ & $0.28300(15)$ & $0.0292(7)$ \\
\hline $\mathrm{H} 4$ & 0.302371 & 0.003680 & 0.270620 & $0.035^{*}$ \\
\hline $\mathrm{C} 12$ & $0.6514(5)$ & $0.40513(15)$ & $0.41843(15)$ & $0.0260(7)$ \\
\hline H12 & 0.783119 & 0.424184 & 0.397451 & $0.031^{*}$ \\
\hline $\mathrm{C} 11$ & $0.5466(5)$ & $0.45039(17)$ & $0.47086(15)$ & $0.0294(7)$ \\
\hline H11 & 0.607818 & 0.499841 & 0.485263 & $0.035^{*}$ \\
\hline $\mathrm{C} 25$ & $0.7808(5)$ & $0.36954(17)$ & $0.09638(15)$ & $0.0279(7)$ \\
\hline $\mathrm{H} 25$ & 0.723985 & 0.422058 & 0.095887 & $0.033^{*}$ \\
\hline $\mathrm{C} 16$ & $1.1623(5)$ & $0.37629(16)$ & $0.25049(15)$ & $0.0257(7)$ \\
\hline H16A & 1.234310 & 0.323625 & 0.252515 & $0.031 *$ \\
\hline H16B & 1.255871 & 0.414955 & 0.276677 & $0.031 *$ \\
\hline $\mathrm{C} 10$ & $0.3465(5)$ & $0.42396(17)$ & $0.50402(16)$ & $0.0293(7)$ \\
\hline
\end{tabular}


supporting information

\begin{tabular}{lllll}
$\mathrm{H} 10$ & 0.276083 & 0.456023 & 0.539681 & $0.035^{*}$ \\
$\mathrm{C} 22$ & $0.9296(5)$ & $0.09975(18)$ & $0.07835(16)$ & $0.0312(7)$ \\
$\mathrm{H} 22$ & 0.908244 & 0.045184 & 0.067111 & $0.037^{*}$ \\
$\mathrm{C} 17$ & $1.1358(5)$ & $0.40255(16)$ & $0.16908(15)$ & $0.0268(7)$ \\
$\mathrm{H} 17 \mathrm{~A}$ & 1.079262 & 0.457682 & 0.167552 & $0.032^{*}$ \\
$\mathrm{H} 17 \mathrm{~B}$ & 1.280575 & 0.402183 & 0.145020 & $0.032^{*}$ \\
$\mathrm{C} 21$ & $1.1245(5)$ & $0.12360(16)$ & $0.11501(16)$ & $0.0306(7)$ \\
$\mathrm{H} 21$ & 1.230368 & 0.084699 & 0.127540 & $0.037^{*}$ \\
\hline
\end{tabular}

Atomic displacement parameters $\left(\AA^{2}\right)$

\begin{tabular}{|c|c|c|c|c|c|c|}
\hline & $U^{11}$ & $U^{22}$ & $U^{33}$ & $U^{12}$ & $U^{13}$ & $U^{23}$ \\
\hline N1 & $0.0244(13)$ & $0.0239(13)$ & $0.0233(13)$ & $-0.0025(11)$ & $-0.0001(11)$ & $0.0016(10)$ \\
\hline $\mathrm{N} 2$ & $0.0235(13)$ & $0.0334(14)$ & $0.0277(12)$ & $0.0037(13)$ & $-0.0039(11)$ & $0.0010(11)$ \\
\hline $\mathrm{C} 18$ & $0.0264(17)$ & $0.0259(16)$ & $0.0194(14)$ & $0.0018(14)$ & $0.0041(13)$ & $0.0033(12)$ \\
\hline $\mathrm{C} 1$ & $0.0216(15)$ & $0.0179(14)$ & $0.0201(14)$ & $0.0003(13)$ & $-0.0036(12)$ & $0.0038(12)$ \\
\hline $\mathrm{C} 8$ & $0.0233(16)$ & $0.0231(15)$ & $0.0184(13)$ & $0.0026(15)$ & $-0.0044(12)$ & $0.0048(12)$ \\
\hline $\mathrm{C} 15$ & $0.0233(16)$ & $0.0198(15)$ & $0.0225(14)$ & $0.0034(14)$ & $-0.0035(12)$ & $-0.0026(12)$ \\
\hline $\mathrm{C} 13$ & $0.0240(15)$ & $0.0208(15)$ & $0.0201(15)$ & $0.0008(13)$ & $-0.0051(12)$ & $0.0042(11)$ \\
\hline C14 & $0.0213(15)$ & $0.0215(15)$ & $0.0190(13)$ & $0.0002(13)$ & $-0.0038(12)$ & $0.0053(11)$ \\
\hline $\mathrm{C} 7$ & $0.0215(15)$ & $0.0280(17)$ & $0.0210(15)$ & $0.0002(13)$ & $-0.0001(12)$ & $0.0068(12)$ \\
\hline C9 & $0.0256(16)$ & $0.0310(18)$ & $0.0230(16)$ & $0.0052(15)$ & $0.0006(13)$ & $0.0040(13)$ \\
\hline C19 & $0.0260(17)$ & $0.0246(16)$ & $0.0177(14)$ & $-0.0010(14)$ & $0.0037(13)$ & $-0.0001(12)$ \\
\hline C6 & $0.0238(16)$ & $0.0259(16)$ & $0.0179(15)$ & $-0.0011(14)$ & $-0.0046(12)$ & $0.0048(11)$ \\
\hline $\mathrm{C} 2$ & $0.0244(15)$ & $0.0245(15)$ & $0.0252(15)$ & $0.0009(14)$ & $0.0032(13)$ & $0.0041(12)$ \\
\hline $\mathrm{C} 5$ & $0.0305(17)$ & $0.0260(16)$ & $0.0257(15)$ & $-0.0071(15)$ & $-0.0019(13)$ & $0.0036(13)$ \\
\hline $\mathrm{C} 20$ & $0.0228(15)$ & $0.0287(16)$ & $0.0253(15)$ & $-0.0012(15)$ & $0.0002(13)$ & $-0.0008(13)$ \\
\hline $\mathrm{C} 24$ & $0.0254(16)$ & $0.0301(16)$ & $0.0193(14)$ & $0.0014(14)$ & $0.0026(13)$ & $0.0027(12)$ \\
\hline $\mathrm{C} 23$ & $0.0252(17)$ & $0.0345(18)$ & $0.0202(14)$ & $-0.0052(15)$ & $-0.0006(12)$ & $-0.0011(13)$ \\
\hline $\mathrm{C} 3$ & $0.0342(18)$ & $0.0203(15)$ & $0.0283(16)$ & $0.0027(15)$ & $0.0020(14)$ & $0.0005(12)$ \\
\hline $\mathrm{C} 4$ & $0.0359(18)$ & $0.0233(16)$ & $0.0284(16)$ & $-0.0070(15)$ & $-0.0023(15)$ & $0.0015(13)$ \\
\hline $\mathrm{C} 12$ & $0.0274(16)$ & $0.0237(15)$ & $0.0270(15)$ & $-0.0007(15)$ & $-0.0010(14)$ & $0.0035(12)$ \\
\hline $\mathrm{C} 11$ & $0.0409(19)$ & $0.0211(15)$ & $0.0262(16)$ & $-0.0018(15)$ & $-0.0046(14)$ & $-0.0021(13)$ \\
\hline $\mathrm{C} 25$ & $0.0339(18)$ & $0.0250(16)$ & $0.0247(15)$ & $0.0030(15)$ & $0.0034(14)$ & $0.0029(12)$ \\
\hline $\mathrm{C} 16$ & $0.0235(15)$ & $0.0214(14)$ & $0.0323(16)$ & $-0.0059(14)$ & $-0.0021(14)$ & $-0.0005(12)$ \\
\hline $\mathrm{C} 10$ & $0.0371(18)$ & $0.0284(17)$ & $0.0223(14)$ & $0.0081(16)$ & $0.0001(15)$ & $-0.0008(12)$ \\
\hline $\mathrm{C} 22$ & $0.0411(19)$ & $0.0263(16)$ & $0.0264(16)$ & $-0.0058(16)$ & $0.0035(15)$ & $-0.0029(13)$ \\
\hline $\mathrm{C} 17$ & $0.0267(16)$ & $0.0208(15)$ & $0.0328(16)$ & $-0.0011(14)$ & $0.0063(14)$ & $0.0026(13)$ \\
\hline $\mathrm{C} 21$ & $0.0352(18)$ & $0.0270(17)$ & $0.0296(16)$ & $0.0055(15)$ & $0.0037(15)$ & $0.0014(13)$ \\
\hline
\end{tabular}

Geometric parameters $\left(\AA,{ }^{\circ}\right)$

\begin{tabular}{llll}
\hline $\mathrm{N} 1-\mathrm{C} 15$ & $1.272(3)$ & $\mathrm{C} 2-\mathrm{H} 2$ & 0.9300 \\
$\mathrm{~N} 1-\mathrm{C} 16$ & $1.468(4)$ & $\mathrm{C} 5-\mathrm{C} 4$ & $1.355(4)$ \\
$\mathrm{N} 2-\mathrm{C} 24$ & $1.371(4)$ & $\mathrm{C} 5-\mathrm{H} 5$ & 0.9300 \\
$\mathrm{~N} 2-\mathrm{C} 25$ & $1.372(3)$ & $\mathrm{C} 20-\mathrm{C} 21$ & $1.380(4)$ \\
$\mathrm{N} 2-\mathrm{H} 2 \mathrm{~A}$ & 0.8600 & $\mathrm{C} 20-\mathrm{H} 20$ & 0.9300 \\
$\mathrm{C} 18-\mathrm{C} 25$ & $1.363(4)$ & $\mathrm{C} 24-\mathrm{C} 23$ & $1.395(4)$
\end{tabular}




\begin{tabular}{|c|c|c|c|}
\hline $\mathrm{C} 18-\mathrm{C} 19$ & $1.444(3)$ & $\mathrm{C} 23-\mathrm{C} 22$ & $1.375(4)$ \\
\hline $\mathrm{C} 18-\mathrm{C} 17$ & $1.499(4)$ & $\mathrm{C} 23-\mathrm{H} 23$ & 0.9300 \\
\hline $\mathrm{C} 1-\mathrm{C} 14$ & $1.411(4)$ & $\mathrm{C} 3-\mathrm{C} 4$ & $1.415(4)$ \\
\hline $\mathrm{C} 1-\mathrm{C} 2$ & $1.426(3)$ & $\mathrm{C} 3-\mathrm{H} 3$ & 0.9300 \\
\hline $\mathrm{C} 1-\mathrm{C} 6$ & $1.436(4)$ & $\mathrm{C} 4-\mathrm{H} 4$ & 0.9300 \\
\hline $\mathrm{C} 8-\mathrm{C} 7$ & $1.387(4)$ & $\mathrm{C} 12-\mathrm{C} 11$ & $1.354(4)$ \\
\hline $\mathrm{C} 8-\mathrm{C} 9$ & $1.420(4)$ & $\mathrm{C} 12-\mathrm{H} 12$ & 0.9300 \\
\hline $\mathrm{C} 8-\mathrm{C} 13$ & $1.440(4)$ & $\mathrm{C} 11-\mathrm{C} 10$ & $1.409(4)$ \\
\hline $\mathrm{C} 15-\mathrm{C} 14$ & $1.479(4)$ & $\mathrm{C} 11-\mathrm{H} 11$ & 0.9300 \\
\hline $\mathrm{C} 15-\mathrm{H} 15$ & 0.9300 & $\mathrm{C} 25-\mathrm{H} 25$ & 0.9300 \\
\hline $\mathrm{C} 13-\mathrm{C} 14$ & $1.417(4)$ & $\mathrm{C} 16-\mathrm{C} 17$ & $1.528(4)$ \\
\hline $\mathrm{C} 13-\mathrm{C} 12$ & $1.429(4)$ & $\mathrm{C} 16-\mathrm{H} 16 \mathrm{~A}$ & 0.9700 \\
\hline $\mathrm{C} 7-\mathrm{C} 6$ & $1.391(4)$ & C16-H16B & 0.9700 \\
\hline $\mathrm{C} 7-\mathrm{H} 7$ & 0.9300 & $\mathrm{C} 10-\mathrm{H} 10$ & 0.9300 \\
\hline $\mathrm{C} 9-\mathrm{C} 10$ & $1.357(4)$ & $\mathrm{C} 22-\mathrm{C} 21$ & $1.398(4)$ \\
\hline C9-H9 & 0.9300 & $\mathrm{C} 22-\mathrm{H} 22$ & 0.9300 \\
\hline $\mathrm{C} 19-\mathrm{C} 20$ & $1.397(4)$ & C17-H17A & 0.9700 \\
\hline $\mathrm{C} 19-\mathrm{C} 24$ & $1.411(4)$ & C17-H17B & 0.9700 \\
\hline $\mathrm{C} 6-\mathrm{C} 5$ & $1.428(4)$ & $\mathrm{C} 21-\mathrm{H} 21$ & 0.9300 \\
\hline $\mathrm{C} 2-\mathrm{C} 3$ & $1.361(4)$ & & \\
\hline $\mathrm{C} 15-\mathrm{N} 1-\mathrm{C} 16$ & $115.2(2)$ & $\mathrm{N} 2-\mathrm{C} 24-\mathrm{C} 23$ & $130.0(3)$ \\
\hline $\mathrm{C} 24-\mathrm{N} 2-\mathrm{C} 25$ & $108.7(2)$ & $\mathrm{N} 2-\mathrm{C} 24-\mathrm{C} 19$ & $107.6(2)$ \\
\hline $\mathrm{C} 24-\mathrm{N} 2-\mathrm{H} 2 \mathrm{~A}$ & 125.7 & $\mathrm{C} 23-\mathrm{C} 24-\mathrm{C} 19$ & $122.4(3)$ \\
\hline $\mathrm{C} 25-\mathrm{N} 2-\mathrm{H} 2 \mathrm{~A}$ & 125.7 & $\mathrm{C} 22-\mathrm{C} 23-\mathrm{C} 24$ & $117.3(3)$ \\
\hline $\mathrm{C} 25-\mathrm{C} 18-\mathrm{C} 19$ & $105.7(3)$ & $\mathrm{C} 22-\mathrm{C} 23-\mathrm{H} 23$ & 121.4 \\
\hline $\mathrm{C} 25-\mathrm{C} 18-\mathrm{C} 17$ & $126.4(2)$ & $\mathrm{C} 24-\mathrm{C} 23-\mathrm{H} 23$ & 121.4 \\
\hline $\mathrm{C} 19-\mathrm{C} 18-\mathrm{C} 17$ & $127.7(3)$ & $\mathrm{C} 2-\mathrm{C} 3-\mathrm{C} 4$ & $121.1(3)$ \\
\hline $\mathrm{C} 14-\mathrm{C} 1-\mathrm{C} 2$ & $123.5(2)$ & $\mathrm{C} 2-\mathrm{C} 3-\mathrm{H} 3$ & 119.5 \\
\hline $\mathrm{C} 14-\mathrm{C} 1-\mathrm{C} 6$ & $119.4(2)$ & $\mathrm{C} 4-\mathrm{C} 3-\mathrm{H} 3$ & 119.5 \\
\hline $\mathrm{C} 2-\mathrm{C} 1-\mathrm{C} 6$ & $117.0(2)$ & $\mathrm{C} 5-\mathrm{C} 4-\mathrm{C} 3$ & $119.5(3)$ \\
\hline $\mathrm{C} 7-\mathrm{C} 8-\mathrm{C} 9$ & $121.2(3)$ & $\mathrm{C} 5-\mathrm{C} 4-\mathrm{H} 4$ & 120.3 \\
\hline $\mathrm{C} 7-\mathrm{C} 8-\mathrm{C} 13$ & $119.4(2)$ & $\mathrm{C} 3-\mathrm{C} 4-\mathrm{H} 4$ & 120.3 \\
\hline $\mathrm{C} 9-\mathrm{C} 8-\mathrm{C} 13$ & $119.4(2)$ & $\mathrm{C} 11-\mathrm{C} 12-\mathrm{C} 13$ & $121.4(3)$ \\
\hline $\mathrm{N} 1-\mathrm{C} 15-\mathrm{C} 14$ & $126.3(3)$ & $\mathrm{C} 11-\mathrm{C} 12-\mathrm{H} 12$ & 119.3 \\
\hline $\mathrm{N} 1-\mathrm{C} 15-\mathrm{H} 15$ & 116.9 & $\mathrm{C} 13-\mathrm{C} 12-\mathrm{H} 12$ & 119.3 \\
\hline $\mathrm{C} 14-\mathrm{C} 15-\mathrm{H} 15$ & 116.9 & $\mathrm{C} 12-\mathrm{C} 11-\mathrm{C} 10$ & $121.2(3)$ \\
\hline $\mathrm{C} 14-\mathrm{C} 13-\mathrm{C} 12$ & $124.0(3)$ & $\mathrm{C} 12-\mathrm{C} 11-\mathrm{H} 11$ & 119.4 \\
\hline $\mathrm{C} 14-\mathrm{C} 13-\mathrm{C} 8$ & $119.0(2)$ & $\mathrm{C} 10-\mathrm{C} 11-\mathrm{H} 11$ & 119.4 \\
\hline $\mathrm{C} 12-\mathrm{C} 13-\mathrm{C} 8$ & $117.0(2)$ & $\mathrm{C} 18-\mathrm{C} 25-\mathrm{N} 2$ & $110.8(2)$ \\
\hline $\mathrm{C} 1-\mathrm{C} 14-\mathrm{C} 13$ & $120.7(2)$ & $\mathrm{C} 18-\mathrm{C} 25-\mathrm{H} 25$ & 124.6 \\
\hline $\mathrm{C} 1-\mathrm{C} 14-\mathrm{C} 15$ & $117.0(2)$ & $\mathrm{N} 2-\mathrm{C} 25-\mathrm{H} 25$ & 124.6 \\
\hline $\mathrm{C} 13-\mathrm{C} 14-\mathrm{C} 15$ & $122.3(2)$ & $\mathrm{N} 1-\mathrm{C} 16-\mathrm{C} 17$ & $110.4(2)$ \\
\hline $\mathrm{C} 8-\mathrm{C} 7-\mathrm{C} 6$ & $122.3(3)$ & $\mathrm{N} 1-\mathrm{C} 16-\mathrm{H} 16 \mathrm{~A}$ & 109.6 \\
\hline $\mathrm{C} 8-\mathrm{C} 7-\mathrm{H} 7$ & 118.8 & $\mathrm{C} 17-\mathrm{C} 16-\mathrm{H} 16 \mathrm{~A}$ & 109.6 \\
\hline $\mathrm{C} 6-\mathrm{C} 7-\mathrm{H} 7$ & 118.8 & $\mathrm{~N} 1-\mathrm{C} 16-\mathrm{H} 16 \mathrm{~B}$ & 109.6 \\
\hline $\mathrm{C} 10-\mathrm{C} 9-\mathrm{C} 8$ & $121.1(3)$ & $\mathrm{C} 17-\mathrm{C} 16-\mathrm{H} 16 \mathrm{~B}$ & 109.6 \\
\hline $\mathrm{C} 10-\mathrm{C} 9-\mathrm{H} 9$ & 119.5 & $\mathrm{H} 16 \mathrm{~A}-\mathrm{C} 16-\mathrm{H} 16 \mathrm{~B}$ & 108.1 \\
\hline
\end{tabular}




\begin{tabular}{|c|c|c|c|}
\hline $\mathrm{C} 8-\mathrm{C} 9-\mathrm{H} 9$ & 119.5 & $\mathrm{C} 9-\mathrm{C} 10-\mathrm{C} 11$ & $119.9(3)$ \\
\hline $\mathrm{C} 20-\mathrm{C} 19-\mathrm{C} 24$ & $118.7(2)$ & $\mathrm{C} 9-\mathrm{C} 10-\mathrm{H} 10$ & 120.1 \\
\hline $\mathrm{C} 20-\mathrm{C} 19-\mathrm{C} 18$ & $134.2(3)$ & $\mathrm{C} 11-\mathrm{C} 10-\mathrm{H} 10$ & 120.1 \\
\hline $\mathrm{C} 24-\mathrm{C} 19-\mathrm{C} 18$ & $107.1(2)$ & $\mathrm{C} 23-\mathrm{C} 22-\mathrm{C} 21$ & $121.5(3)$ \\
\hline $\mathrm{C} 7-\mathrm{C} 6-\mathrm{C} 5$ & $121.5(2)$ & $\mathrm{C} 23-\mathrm{C} 22-\mathrm{H} 22$ & 119.3 \\
\hline $\mathrm{C} 7-\mathrm{C} 6-\mathrm{C} 1$ & $119.2(2)$ & $\mathrm{C} 21-\mathrm{C} 22-\mathrm{H} 22$ & 119.3 \\
\hline $\mathrm{C} 5-\mathrm{C} 6-\mathrm{C} 1$ & $119.3(2)$ & $\mathrm{C} 18-\mathrm{C} 17-\mathrm{C} 16$ & $112.2(2)$ \\
\hline $\mathrm{C} 3-\mathrm{C} 2-\mathrm{C} 1$ & $121.7(3)$ & $\mathrm{C} 18-\mathrm{C} 17-\mathrm{H} 17 \mathrm{~A}$ & 109.2 \\
\hline $\mathrm{C} 3-\mathrm{C} 2-\mathrm{H} 2$ & 119.2 & $\mathrm{C} 16-\mathrm{C} 17-\mathrm{H} 17 \mathrm{~A}$ & 109.2 \\
\hline $\mathrm{C} 1-\mathrm{C} 2-\mathrm{H} 2$ & 119.2 & $\mathrm{C} 18-\mathrm{C} 17-\mathrm{H} 17 \mathrm{~B}$ & 109.2 \\
\hline $\mathrm{C} 4-\mathrm{C} 5-\mathrm{C} 6$ & $121.4(3)$ & $\mathrm{C} 16-\mathrm{C} 17-\mathrm{H} 17 \mathrm{~B}$ & 109.2 \\
\hline $\mathrm{C} 4-\mathrm{C} 5-\mathrm{H} 5$ & 119.3 & $\mathrm{H} 17 \mathrm{~A}-\mathrm{C} 17-\mathrm{H} 17 \mathrm{~B}$ & 107.9 \\
\hline $\mathrm{C} 6-\mathrm{C} 5-\mathrm{H} 5$ & 119.3 & $\mathrm{C} 20-\mathrm{C} 21-\mathrm{C} 22$ & $121.1(3)$ \\
\hline $\mathrm{C} 21-\mathrm{C} 20-\mathrm{C} 19$ & $119.1(3)$ & $\mathrm{C} 20-\mathrm{C} 21-\mathrm{H} 21$ & 119.5 \\
\hline $\mathrm{C} 21-\mathrm{C} 20-\mathrm{H} 20$ & 120.5 & $\mathrm{C} 22-\mathrm{C} 21-\mathrm{H} 21$ & 119.5 \\
\hline $\mathrm{C} 19-\mathrm{C} 20-\mathrm{H} 20$ & 120.5 & & \\
\hline $\mathrm{C} 16-\mathrm{N} 1-\mathrm{C} 15-\mathrm{C} 14$ & $-179.0(2)$ & $\mathrm{C} 7-\mathrm{C} 6-\mathrm{C} 5-\mathrm{C} 4$ & $177.9(3)$ \\
\hline $\mathrm{C} 7-\mathrm{C} 8-\mathrm{C} 13-\mathrm{C} 14$ & $1.7(3)$ & $\mathrm{C} 1-\mathrm{C} 6-\mathrm{C} 5-\mathrm{C} 4$ & $-1.0(4)$ \\
\hline $\mathrm{C} 9-\mathrm{C} 8-\mathrm{C} 13-\mathrm{C} 14$ & $-179.8(2)$ & $\mathrm{C} 24-\mathrm{C} 19-\mathrm{C} 20-\mathrm{C} 21$ & $-1.1(4)$ \\
\hline $\mathrm{C} 7-\mathrm{C} 8-\mathrm{C} 13-\mathrm{C} 12$ & $-177.6(2)$ & $\mathrm{C} 18-\mathrm{C} 19-\mathrm{C} 20-\mathrm{C} 21$ & $177.4(3)$ \\
\hline $\mathrm{C} 9-\mathrm{C} 8-\mathrm{C} 13-\mathrm{C} 12$ & $0.9(3)$ & $\mathrm{C} 25-\mathrm{N} 2-\mathrm{C} 24-\mathrm{C} 23$ & $178.3(3)$ \\
\hline $\mathrm{C} 2-\mathrm{C} 1-\mathrm{C} 14-\mathrm{C} 13$ & $176.9(2)$ & $\mathrm{C} 25-\mathrm{N} 2-\mathrm{C} 24-\mathrm{C} 19$ & $-0.1(3)$ \\
\hline $\mathrm{C} 6-\mathrm{C} 1-\mathrm{C} 14-\mathrm{C} 13$ & $-0.5(4)$ & $\mathrm{C} 20-\mathrm{C} 19-\mathrm{C} 24-\mathrm{N} 2$ & $179.1(2)$ \\
\hline $\mathrm{C} 2-\mathrm{C} 1-\mathrm{C} 14-\mathrm{C} 15$ & $-5.9(4)$ & $\mathrm{C} 18-\mathrm{C} 19-\mathrm{C} 24-\mathrm{N} 2$ & $0.2(3)$ \\
\hline $\mathrm{C} 6-\mathrm{C} 1-\mathrm{C} 14-\mathrm{C} 15$ & $176.6(2)$ & $\mathrm{C} 20-\mathrm{C} 19-\mathrm{C} 24-\mathrm{C} 23$ & $0.6(4)$ \\
\hline $\mathrm{C} 12-\mathrm{C} 13-\mathrm{C} 14-\mathrm{C} 1$ & $178.7(2)$ & $\mathrm{C} 18-\mathrm{C} 19-\mathrm{C} 24-\mathrm{C} 23$ & $-178.3(3)$ \\
\hline $\mathrm{C} 8-\mathrm{C} 13-\mathrm{C} 14-\mathrm{C} 1$ & $-0.5(4)$ & $\mathrm{N} 2-\mathrm{C} 24-\mathrm{C} 23-\mathrm{C} 22$ & $-177.8(3)$ \\
\hline $\mathrm{C} 12-\mathrm{C} 13-\mathrm{C} 14-\mathrm{C} 15$ & $1.8(4)$ & $\mathrm{C} 19-\mathrm{C} 24-\mathrm{C} 23-\mathrm{C} 22$ & $0.3(4)$ \\
\hline $\mathrm{C} 8-\mathrm{C} 13-\mathrm{C} 14-\mathrm{C} 15$ & $-177.5(2)$ & $\mathrm{C} 1-\mathrm{C} 2-\mathrm{C} 3-\mathrm{C} 4$ & $0.1(4)$ \\
\hline $\mathrm{N} 1-\mathrm{C} 15-\mathrm{C} 14-\mathrm{C} 1$ & $147.8(3)$ & $\mathrm{C} 6-\mathrm{C} 5-\mathrm{C} 4-\mathrm{C} 3$ & $-0.3(4)$ \\
\hline $\mathrm{N} 1-\mathrm{C} 15-\mathrm{C} 14-\mathrm{C} 13$ & $-35.1(4)$ & $\mathrm{C} 2-\mathrm{C} 3-\mathrm{C} 4-\mathrm{C} 5$ & $0.8(4)$ \\
\hline $\mathrm{C} 9-\mathrm{C} 8-\mathrm{C} 7-\mathrm{C} 6$ & $179.6(2)$ & $\mathrm{C} 14-\mathrm{C} 13-\mathrm{C} 12-\mathrm{C} 11$ & $-179.9(3)$ \\
\hline $\mathrm{C} 13-\mathrm{C} 8-\mathrm{C} 7-\mathrm{C} 6$ & $-1.9(4)$ & $\mathrm{C} 8-\mathrm{C} 13-\mathrm{C} 12-\mathrm{C} 11$ & $-0.6(4)$ \\
\hline $\mathrm{C} 7-\mathrm{C} 8-\mathrm{C} 9-\mathrm{C} 10$ & $177.9(2)$ & $\mathrm{C} 13-\mathrm{C} 12-\mathrm{C} 11-\mathrm{C} 10$ & $-0.1(4)$ \\
\hline $\mathrm{C} 13-\mathrm{C} 8-\mathrm{C} 9-\mathrm{C} 10$ & $-0.6(4)$ & $\mathrm{C} 19-\mathrm{C} 18-\mathrm{C} 25-\mathrm{N} 2$ & $0.3(3)$ \\
\hline $\mathrm{C} 25-\mathrm{C} 18-\mathrm{C} 19-\mathrm{C} 20$ & $-178.9(3)$ & $\mathrm{C} 17-\mathrm{C} 18-\mathrm{C} 25-\mathrm{N} 2$ & $-175.1(2)$ \\
\hline $\mathrm{C} 17-\mathrm{C} 18-\mathrm{C} 19-\mathrm{C} 20$ & $-3.6(5)$ & $\mathrm{C} 24-\mathrm{N} 2-\mathrm{C} 25-\mathrm{C} 18$ & $-0.2(3)$ \\
\hline $\mathrm{C} 25-\mathrm{C} 18-\mathrm{C} 19-\mathrm{C} 24$ & $-0.3(3)$ & $\mathrm{C} 15-\mathrm{N} 1-\mathrm{C} 16-\mathrm{C} 17$ & $111.3(3)$ \\
\hline $\mathrm{C} 17-\mathrm{C} 18-\mathrm{C} 19-\mathrm{C} 24$ & $175.0(3)$ & $\mathrm{C} 8-\mathrm{C} 9-\mathrm{C} 10-\mathrm{C} 11$ & $-0.1(4)$ \\
\hline $\mathrm{C} 8-\mathrm{C} 7-\mathrm{C} 6-\mathrm{C} 5$ & $-178.2(2)$ & $\mathrm{C} 12-\mathrm{C} 11-\mathrm{C} 10-\mathrm{C} 9$ & $0.5(4)$ \\
\hline $\mathrm{C} 8-\mathrm{C} 7-\mathrm{C} 6-\mathrm{C} 1$ & $0.8(4)$ & $\mathrm{C} 24-\mathrm{C} 23-\mathrm{C} 22-\mathrm{C} 21$ & $-0.7(4)$ \\
\hline $\mathrm{C} 14-\mathrm{C} 1-\mathrm{C} 6-\mathrm{C} 7$ & $0.5(4)$ & $\mathrm{C} 25-\mathrm{C} 18-\mathrm{C} 17-\mathrm{C} 16$ & $115.4(3)$ \\
\hline $\mathrm{C} 2-\mathrm{C} 1-\mathrm{C} 6-\mathrm{C} 7$ & $-177.2(2)$ & $\mathrm{C} 19-\mathrm{C} 18-\mathrm{C} 17-\mathrm{C} 16$ & $-59.1(4)$ \\
\hline $\mathrm{C} 14-\mathrm{C} 1-\mathrm{C} 6-\mathrm{C} 5$ & $179.4(2)$ & $\mathrm{N} 1-\mathrm{C} 16-\mathrm{C} 17-\mathrm{C} 18$ & $-55.6(3)$ \\
\hline $\mathrm{C} 2-\mathrm{C} 1-\mathrm{C} 6-\mathrm{C} 5$ & $1.8(4)$ & $\mathrm{C} 19-\mathrm{C} 20-\mathrm{C} 21-\mathrm{C} 22$ & $0.7(4)$ \\
\hline $\mathrm{C} 14-\mathrm{C} 1-\mathrm{C} 2-\mathrm{C} 3$ & $-178.9(3)$ & $\mathrm{C} 23-\mathrm{C} 22-\mathrm{C} 21-\mathrm{C} 20$ & $0.2(4)$ \\
\hline $\mathrm{C} 6-\mathrm{C} 1-\mathrm{C} 2-\mathrm{C} 3$ & $-1.3(4)$ & & \\
\hline
\end{tabular}


Hydrogen-bond geometry $\left(\AA,{ }^{\circ}\right)$

$C g 3, C g 4$ and $C g 5$ are the centroids rings $\mathrm{C} 1 / \mathrm{C} 6-\mathrm{C} 8 / \mathrm{C} 13 / \mathrm{C} 14, \mathrm{C} 8-\mathrm{C} 13$ and $\mathrm{C} 19-\mathrm{C} 24$, respectively.

\begin{tabular}{lllll}
\hline$D-\mathrm{H} \cdots A$ & $D-\mathrm{H}$ & $\mathrm{H} \cdots A$ & $D \cdots A$ & $D-\mathrm{H} \cdots A$ \\
\hline $\mathrm{C} 12-\mathrm{H} 12 \cdots \mathrm{N} 1$ & 0.93 & 2.36 & $2.9845(2)$ & 124 \\
$\mathrm{C} 2-\mathrm{H} 2 \cdots C g 5$ & 0.93 & 2.77 & $3.5505(2)$ & 142 \\
$\mathrm{~N} 2-\mathrm{H} 2 A \cdots C g 5^{\mathrm{i}}$ & 0.86 & 2.59 & $3.1855(2)$ & 127 \\
$\mathrm{C} 7-\mathrm{H} 7 \cdots C g 4^{\mathrm{ii}}$ & 0.93 & 2.75 & $3.5777(2)$ & 148 \\
$\mathrm{C} 9-\mathrm{H} 9 \cdots C g 3^{\mathrm{ii}}$ & 0.93 & 2.73 & $3.5077(2)$ & 142 \\
$\mathrm{C} 16-\mathrm{H} 16 A \cdots C g 3^{\mathrm{iii}}$ & 0.97 & 2.86 & $3.5375(2)$ & 128
\end{tabular}

Symmetry codes: (i) $x-1 / 2,-y+1 / 2,-z$; (ii) $x-1 / 2,-y+1 / 2,-z+1$; (iii) $x+1, y, z$. 